\title{
Design of Digital Low Pass IIR Filter using Real Coded Genetic Algorithm
}

\author{
Bhagyashree K. Jagtap \\ KIT's College of Engineering, Kolhapur, \\ Maharashtra, India
}

\author{
Mahesh S. Chavan \\ KIT's College of Engineering, Kolhapur, \\ Maharashtra, India
}

\begin{abstract}
This paper presents the design method for Infinite Impulse Response (IIR) digital filter with the use of Real Coded Genetic Algorithm (RGA/RCGA). Generally, nonlinear and multimodal error surface is observed in case of digital IIR filter. Hence, to avoid problems such as local minima, global optimization techniques are required. This paper gives effective method to design digital IIR filters. This paper finds the optimum coefficients of IIR digital filter by using RGA. RGA works on real numbers and is a powerful global optimization algorithm. To obtain minimum transition band, this designing of low pass IIR digital filter is proposed. It is observed that the calculated values are more optimal than those which are obtained by FDA tool available for design of filter in MATLAB. The improved mean-square-error (MSE) is observed in simulation results for example taken.
\end{abstract}

\section{General Terms}

Digital filter design, optimization algorithm, Frequency response, filter coefficients

\section{Keywords}

Infinite Impulse Response (IIR) filter, Real Coded Genetic Algorithm (RGA/RCGA), Stability

\section{INTRODUCTION}

In last few decades the science and technology is getting more and more advanced. This advancement is touching our lives in all aspects. Specially talking about Bio-medical field, it has become very much wide spread, advanced, fast and accurate, with handy equipments, easy to use testing devices and readable output devices. When we talk about progress of biomedical field, one should mention that, signal processing plays most important part in it. The biomedical signals like ECG, EKG, EMG, etc. where signal processing is very important task, and it should be done very carefully, because wrong analysis may cause wrong diagnosis, which should not happen. Hence for accurate analysis of such signals, signal accuracy should be maintained, for this signal must be filtered, prior to its use for analysis purpose.

Filters are mainly classified of two types. One of them is called analog filters and other type is called digital filters. Analog filters are made by using discrete components namely as, resistor, capacitor, inductor and op-amp. Main drawback of analog filters is the component tolerance, large hardware size, poor accuracy. These are overcome in digital filters as it performs only mathematical operations on sampled data or discrete signals. The digital filter can be designed with the help of processor in computer or the DSP kit. One of the most powerful tools of DSP is Digital filter. Digital filters give best specifications of performance. On contrary it would be very hard to obtain the similar specifications with analog implementation. Further these digital filters are broadly divided in two classes, depending on the form of impulse response of the system, as Finite Impulse Response (FIR) and Infinite Impulse Response (IIR). In FIR filter output value depends upon present input and present output. But, in IIR filter output value depends upon present output and past output, as well. Therefore, FIR is called non-recursive filter, on the contrary, IIR is called recursive filter. Since IIR filter needs present and past output values, it requires large memory to store all this data. But, the FIR needs lesser memory, as its output depends on only present input and present output. Most importantly IIR filter meets desired specifications, with lesser ripple content in pass band and stop band, sharp transition band, with low filter order than that with FIR. We can say, properly designed IIR filter can give almost ideal response compared to that of FIR filter.

While designing a digital IIR filter, we must strictly follow some constraints in order to meet the overall design criteria satisfactorily: 1)Filter order must be as low as possible. 2)The filter must be the stable one i.e. its poles must lie inside the unit circle. 3)The predefined tolerance settings must be fulfilled by the filter which are determined by reducing the error magnitudes of ripple of pass band and stop band. But in optimization process, it's very difficult to satisfy the final digital filter design as to obtain the desired filter performance with these constraints. The optimization is applied for finding the optimal coefficients of the digital IIR filter, so that we get filter response approximately closely to the desired response of the filter.

As compared to FIR filter, an IIR filter requires less computation for the same performance. IIR digital filters are proved very much useful in many applications. Mainly in the applications, which needs high selectivity and which requires efficient processing of discrete signals. A reliable designing method based on a global search process is needed for designing IIR filter to eliminate the problem of multi-modal error surface existing with IIR filter. The main task in IIR type digital filter designing is to obtain approximately an ideal frequency response from a stable IIR type digital filter by the use of some design criterion. For implementing optimization technique under some design criteria, different optimization methods have been used where ripple magnitudes (tolerances) of pass-band as well as stop-band and mean square-error are used for measuring performance [1-3]. Due to non-linear and multimodal nature of error surface of IIR filters, gradientbased design methods may easily get stuck in the local minima of error surface [4]. Therefore, researchers have developed filter designing methods based on modern heuristics optimization algorithms such as genetic algorithms [5]. Genetic algorithm (GA) and Evolutionary Programming (EP) are general purpose search algorithms which make use of 
evolutionary ideas of natural selection and genetic dynamics [6-10] to find the global optimal solution of the problem. The ability of genetic algorithms to combine both exploration and exploitation in an optimal way is one of their main assets [11]. In many multidimensional and multimodal engineering design problems, the GA has been used as a robust and proficient search method. GA requires less iterative computational equations as compared to traditional search algorithms like calculus based searches, random searches, dynamic programming and gradient methods, but convergence time taken to reach the solution is more. The Real Coded Genetic Algorithm (RCGA) is a GA which uses real coding technique. There are various existing coding schemes used for coding of search space solutions, but out of those techniques, real coding technique particularly seems natural when handling optimization problems. Whenever a parameter is binary coded, there is always risk of getting reduced precision level, because it does not represent parameter values which produces the best solution values. The final local tuning capability of a binary coded genetic algorithm is improved in the RCGA, which is needed the most for high precision optimization problems. Real-coding of the genes eliminates concern that precision is not adequate for representing good search space values. The magnitude response of IIR filter is more important as compared to phase response, in real time applications. The intent of this paper is to apply a RCGA for the design of stable digital IIR filter. The RCGA approach is used for optimizing the filter coefficients to achieve minimum approximation error criterion.

The further part of paper is organized as follows. The Section 2 describes the IIR filter formulation. The implementation of RCGA algorithm for designing the optimal digital IIR filter is described in Section 3. In Section 4, the simulation results and discussions of the proposed RCGA algorithm has been evaluated and there results are compared with the results of ideal filter and with the filter without optimization. At last, the conclusions are outlined in Section 5.

\section{IIR FILTER FORMULATION}

This section is to discuss the designing of IIR filter. The input-output relation can be expressed with the following difference equation

$$
y(p)+\sum_{k=1}^{n} a_{k} y(p-k)=\sum_{k=0}^{m} b_{k} x(p-k) \ldots \ldots \ldots \ldots
$$

Where $x(p)$ and $y(p)$ are the filter's input and output, respectively. Here, $\mathrm{n}(>\mathrm{m})$ is the filter's order. Assuming the coefficient $\mathrm{a}_{0}=0$, the transfer function of the IIR filter can be expressed as

$$
H(z)=\frac{B(z)}{A(z)}=\frac{\sum_{k=0}^{m} b_{k} z^{-k}}{1+\sum_{k=1}^{n} a_{k} z^{-k}}
$$

Let, $\mathrm{z}=\mathrm{e}^{\mathrm{j} \Omega}$. Then frequency response of the IIR filter become

$$
H(\Omega)=\frac{\sum_{k=0}^{m} b_{k} e^{-j k \Omega}}{1+\sum_{k=1}^{n} a_{k} e^{-j k \Omega}}
$$

Or,

$$
\begin{aligned}
& H(\Omega)=\frac{Y(\Omega)}{X(\Omega)} \\
& =\frac{b_{0}+b_{1} e^{-j \Omega}+b_{2} e^{-j 2 \Omega}+\cdots+b_{m} e^{-j m \Omega}}{1+a_{1} e^{-j \Omega}+a_{2} e^{-j 2 \Omega}+\cdots+a_{m} e^{-j n \Omega}} \cdots
\end{aligned}
$$

Where, $\Omega=2 \Pi\left(\frac{f}{f_{s}}\right)$ in $[0, \Pi]$ is the digital frequency, $f$ is the analog frequency and $f_{s}$ is the sampling frequency.

Here, we need to design $6^{\text {th }}$ order $100 \mathrm{~Hz}$ Low Pass IIR digital filter with following specifications:

$\mathrm{W}_{\mathrm{p}}=100 \mathrm{~Hz}$
$\mathrm{~W}_{\mathrm{s}}=110 \mathrm{~Hz}$
$\mathrm{R}_{\mathrm{p}}=2$
$\mathrm{R}_{\mathrm{s}}=40$

Sampling Frequency $=1000 \mathrm{~Hz}$

\section{IMPLEMENTATION OF RCGA ALGORITHM}

In Real Coded Genetic Algorithm, the parameters are represented without coding. Hence, it makes the solutions representation very much similar to the natural formulation of a problem. In the RCGA algorithm, the recombination operator and mutation operator needs to be designed to work with the real parameters only. It is possible to use large domain (even unknown domain) for variables, since the algorithm makes use of real-parameter. Its capacity to exploit the graduality of the functions with the help of continuous variables, is its another advantage. In the real-coded genetic algorithm, corresponding to the design variables, real numbers are coded in a finite length of string called as chromosome. For the crossover operation, in the binary-coded GA, the operator makes cuts between genes, which causes change in the value of the goal function variables, but in real-coded GA operator interchanges just the variables between individuals.

In Real Coded Genetic Algorithm, at a time it searches for many points in its search space, and it goes on narrowing focus of search continuously, in the area of best performance observed previously. We can enlist the basic elements of RCGA as reproduction, selection, crossover, and mutation. The individuals with higher fitness gets selected from existing population, for reproduction operation. Crossover is the operation in which two individuals are selected randomly from mating pool and then crossover is done by using mathematical relations. In GA, Mutation is important part, as it prevents the search to go close to local optimum of the search space.

\subsection{Real Coded Genetic Algorithm:}

1. Initialize the chromosome strings of population with real coding, each having equal number of numerator and denominator filter coefficients $b_{k}$ and $a_{k}$, respectively.

2. Evaluate of error fitness values of each and every member of population.

3. Select of the strings in order of increasing error fitness values from the minimum value.

4. Select the highly fit members from population as parents by using roulette wheel selection. Now produce offsprings according to their fitness value.

5. Create new member by mating current offspring's. Applying crossover and mutation operators so that variations gets introduced and forms new member.

6. Replace existing member with new member, after competition and selection. 
7. Go to Step 2, and repeat the process until it meets the termination criterion.

\section{STOP.}

The design specifications of IIR LP filter are given in Table 1 . While using the Real Coded genetic algorithm some control parameters are required. Here, the control parameters of RCGA used in this paper are given in Table 2. These control parameters are adopted after some trials so that we get optimum output from filter.

\section{SIMULATION RESULTS AND DISCUSIONS}

As shown in figure 1. there are three graphs together in one plot. Out of these three the upper plot is a frequency response plot obtained with the IIR filter designed without using RCGA technique, shown in green color, along with Ideal response of IIR filter for similar cutoff frequency, which is shown in blue color. Here, Y-axis represents normalised gain (o to 1) of filter and $\mathrm{X}$-axis represents samples of frequency $\left(500 \mathrm{~Hz}\right.$ is sampled at $1000^{\text {th }}$ point). The middle plot is a frequency response of the IIR type digital filter designed with RCGA algorithm shown in green color, and is compared with Ideal response of IIR filter for similar cutoff frequency, which is shown in blue color. Here, similar to upper plot, Y-axis represents normalised gain (o to 1) of filter and $\mathrm{X}$-axis represents samples of frequency $(500 \mathrm{~Hz}$ is placed at the place of $1000^{\text {th }}$ point). If we observe these two plots, we can get that the ripples present in the Actual response have got decreased to very much extend in RCGA optimized response. Hence, optimization in percentage has become 79.6875. So it indicates that error function has got improved very much. The third plot in the figure is pole-zero plot of optimized filter whose coefficirents are given below. The plot shows that all the poles and zeros lie inside the unit circle,hence the filter is stable in nature.

The realized model of optimized IIR LP filter, shown in figure 2 , is obtained from FDA tool available in MATLAB. Percentage of optimization is calculated from difference of two fitness values obtained from the filter without optimization and with optimization divided by fitness value without optimization.

$\begin{array}{lcccccc}\text { RGA }[\mathrm{a} \text { b] }=-9.5186 & 9.9928 & 4.4815 & -2.3057 & - \\ 5.0577 & -0.55082 & 2.5746 & -8.8527 & 0.20212 & -8.8373 \\ -8.7616 & 1.4766 & 9.5601 & 6.5658 & & \end{array}$

RGA Optimization \%:=79.6875

\section{CONCLUSION}

This paper proposes a RCGA approach for optimization of digital IIR filters considering multiple objectives like getting good stability, reduced ripple in pass band. On the basis of results obtained for the design of digital IIR filter, it can be concluded that RCGA is a robust algorithm. More focus is required, on reducing the transition width of the filter, while designing the filter. RCGA can design a digital filter of any kind. This design provides good magnitude and phase response with lesser order of the filter. Concluding, Simulation studies show that the proposed method is accurate, robust and an efficient optimizer for digital IIR filter design.
Table 1: Design specifications of IIR Low Pass Filter

\begin{tabular}{|l|l|l|l|l|}
\hline $\begin{array}{l}\text { Type } \\
\text { of } \\
\text { Filter }\end{array}$ & $\begin{array}{l}\text { Pass } \\
\text { band } \\
\text { ripple } \\
\left(\delta_{\mathrm{p}}\right)\end{array}$ & $\begin{array}{l}\text { Stop } \\
\text { band } \\
\text { ripple } \\
\left(\delta_{\mathrm{s}}\right)\end{array}$ & $\begin{array}{l}\text { Pass band } \\
\text { normalized } \\
\text { edge frequency } \\
\left(\omega_{\mathrm{p}}\right)\end{array}$ & $\begin{array}{l}\text { Stop band } \\
\text { normalized } \\
\text { edge frequency } \\
\left(\omega_{\mathrm{s}}\right)\end{array}$ \\
\hline $\begin{array}{l}\text { Low } \\
\text { Pass }\end{array}$ & 2 & 40 & 0.100 & 0.110 \\
\hline
\end{tabular}

Table 2: Control parameters of RCGA

\begin{tabular}{|c|c|}
\hline Parameters & RGA \\
\hline Population Size & 100 \\
\hline Iteration Cycle & 50 \\
\hline Crossover \% & 70 \\
\hline Mutation Rate & 0.1 \\
\hline Mutation & Uniform \\
\hline
\end{tabular}
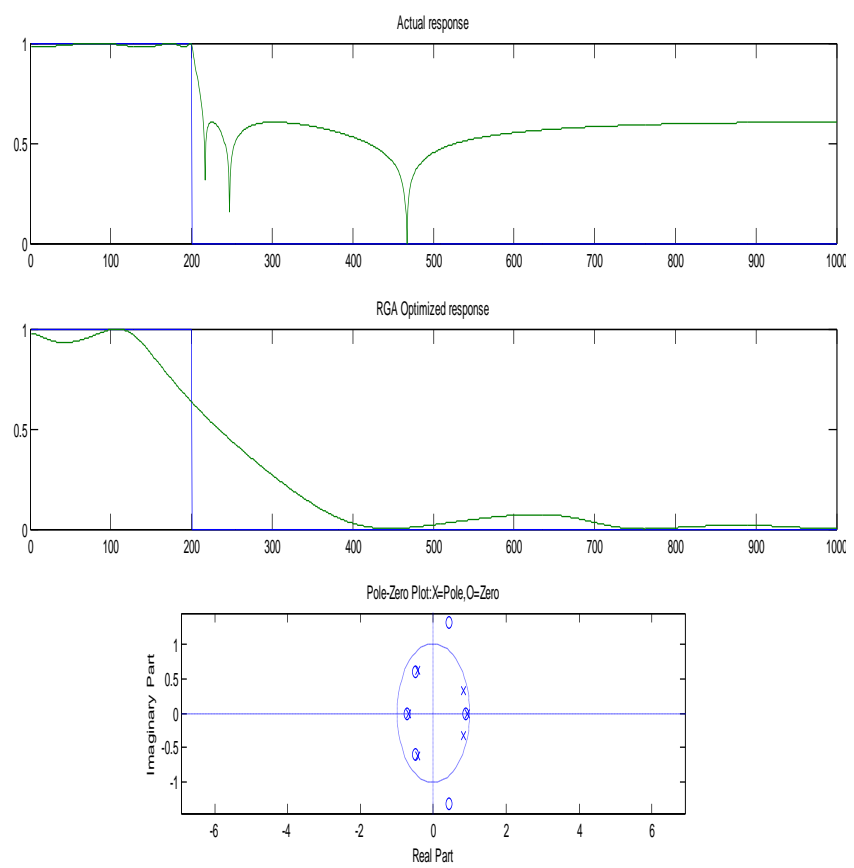

Figure 1: Filter Response and Pole Zero plot before and after application of Real Coded Genetic Algorithm 


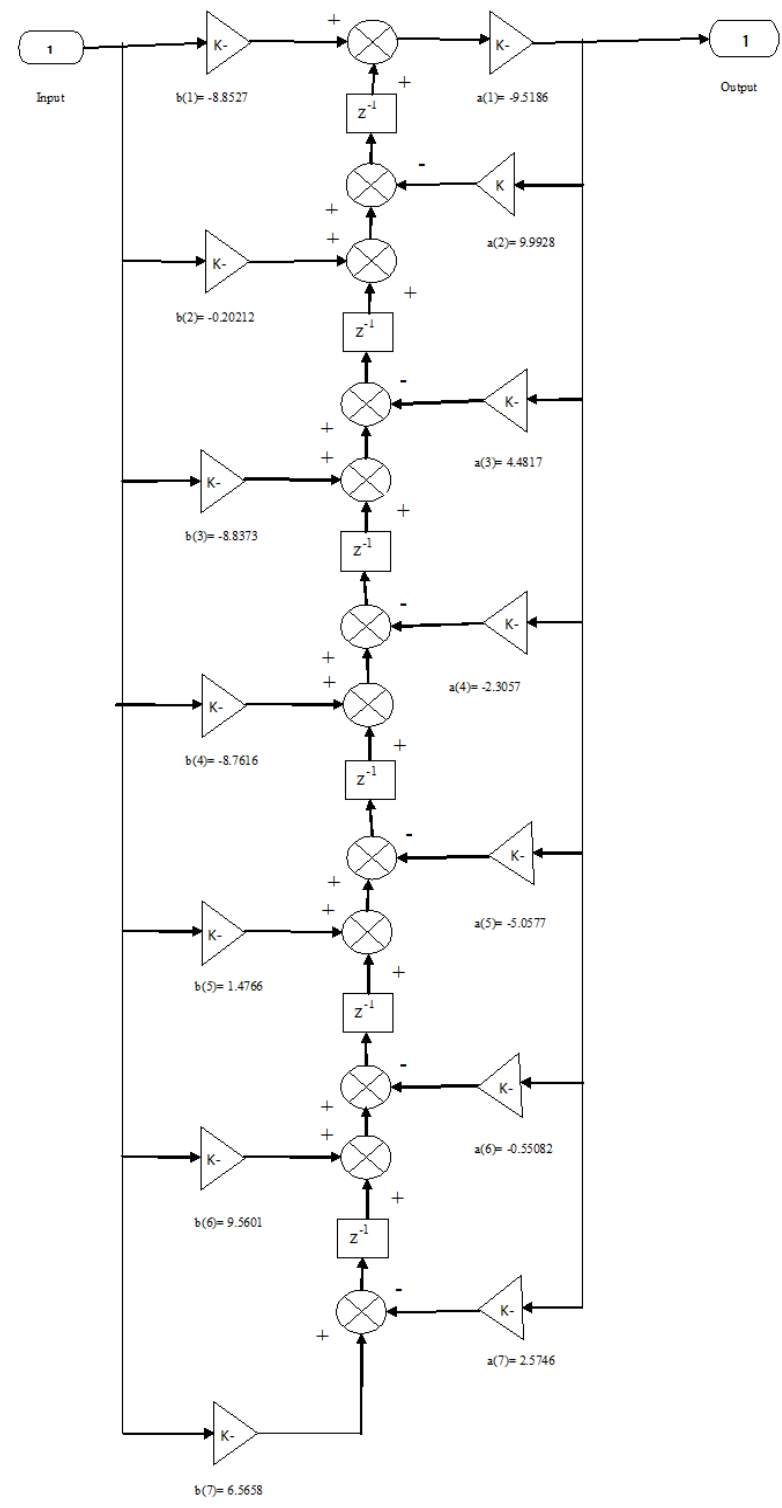

Figure 2: Realized Model of Low Pass Filter after Optimization

\section{REFERENCES}

[1] W.S. Lu, W.-S. Lu, S. Pei, and C. Tseng, "A weighted least-squares method for the design of stable 1-D and 2D IIR digital filters," IEEE Trans. Signal Process., vol. 46, no. 1, pp. 1-10, Jan. 1998.

[2] C. Tseng and S. Lee, "Minimax design of stable IIR digital filter with prescribed magnitude and phase responses," IEEE Trans. Circuits Syst. I, Reg. Papers, vol. 49, no. 4, pp. 547-551, April 2002.

[3] C. Tseng, "Design of stable IIR digital filter based on least p-power error criterion," IEEE Trans. Circuits Syst. I, Reg. Papers, vol. 51, no.9, pp. 1879-1888, Sept. 2004.

[4] S.H. Ling, H.H.C. Iu, F.H.F. Leung, and K.Y. Chan, "Improved hybrid particle swarm optimized wavelet neural network for modeling the development of fluid dispensing for electronic packaging," IEEE Trans. Ind. Electron., vol. 55, no. 9, pp. 3447-3460, Sept. 2008.

[5] K.S. Tang, K.F. Man, S. Kwong and Z.F. Liu, "Design and optimization of IIR filter structure using hierarchal genetic algorithms, "IEEE Transactions on Industrial Electronics, vol. 45, no. 3, pp. 481-487, June 1998.

[6] D.E. Goldberg, "Genetic algorithms in search, optimization, and machine learning," Addison-Wesley: New York, 1989.

[7] R. Chiong and O.K. Beng, "A Comparison between genetic algorithms and evolutionary programming based on cutting ctock problem," Engineering Letters, vol. 14, issue 1, Feb. 2007, pp72-77.

[8] Terry Jones, Stephanie Forrest, "Genetic algorithms and heuristic search," SFI working paper:1995-02-021

[9] Mehrdad Dianati, Inop Song and Mark Treiber, "An introduction to genetic algorithms and evolution strategies," University of Waterloo, Ontario, N2L 3G1, Canada.

[10] Melanie Mitchell, "Genetic algorithms: an overview," Santa Fe Institute, Comlexity, 1 (1) 31-39, 1995, Adapted from An Introduction to Genetic Algorithms, Chapter 1, MIT Press.

[11] T.A. El-Mihoub, A.A. Hopgood, L.S Nolle and A. Battersby, "Hybrid genetic algorithms: a Review," Engineering Letters, vol. 13, issue 2, Aug. 2006, pp124137. 